\title{
Erratum to: Overexpression of Renal Tumor Antigen Is Associated with Tumor Invasion and Poor Prognosis of Hepatocellular Carcinoma
}

Hyung Jin Cha, BS ${ }^{1}$, Jongmin Kim, PhD $^{2}$, Sun Mi Hong, BS ${ }^{1}$, Seok Joo Hong, PhD ${ }^{2}$, Jun Ho Park, BS ${ }^{2}$, Eung-Sam Kim, MS ${ }^{3}$, Hee-Jung Wang, $\mathrm{MD}^{4}$, Yoon Jung Choi, $\mathrm{MD}^{5}$, In-Gu Do, $\mathrm{MD}^{6}$, Jae Won Joh, $\mathrm{MD}^{7}$, Dae Shick Kim, MD, $\mathbf{P h D}^{8}$, and Kwan Yong Choi, $\mathbf{P h D}^{1,3}$

${ }^{1}$ Department of Life Science, Pohang University of Science and Technology, Pohang, Korea; ${ }^{2}$ Cbs Bioscience Inc., Daejeon, Korea; ${ }^{3}$ School of Interdisciplinary Bioscience and Bioengineering, Pohang University of Science and Technology, Pohang, Korea; ${ }^{4}$ Department of Surgery, Ajou University School of Medicine, Suwon, Korea; ${ }^{5}$ Department of Pathology, National Health Insurance Corporation Ilsan Hospital, Goyang, Gyeonggi, Korea; ${ }^{6}$ Experimental Pathology Center, Samsung Cancer Research Institute, Samsung Medical Center, Seoul, Korea; ${ }^{7}$ Department of Surgery, Samsung Medical Center, Sungkyunkwan University School of Medicine, Seoul, Korea; ${ }^{8}$ Department of Pathology, Samsung Medical Center, Sungkyunkwan University School of Medicine, Seoul, Korea

\section{ERRATUM TO: ANN SURG ONCOL}

\section{DOI 10.1245/S10434-011-1856-3}

In the original publication, the correct correspondence information for author D. S. Kim was not included. Both D. S. Kim (oncorkim@skku.edu) and K. Y. Choi (kchoi@postech.ac.kr) are corresponding authors for this paper.

The online version of the original article can be found under doi:10.1245/s10434-011-1856-3.

(C) Society of Surgical Oncology 2011

First Received: 27 September 2011;

Published Online: 12 October 2011

D. S. Kim, MD, PhD

e-mail: oncorkim@skku.edu

K. Y. Choi, PhD

e-mail: kchoi@postech.ac.kr 